\title{
Structural exploring multi-component equilibrium in biological systems
}

\author{
Hironari Kamikubo $^{1}$, Yoichi Yamazaki ${ }^{1}$ \\ ${ }^{1}$ Nara Institute Of Science And Technology, Ikoma, Japan \\ E-mail: kamikubo@ms.naist.jp
}

Various protein molecules concert with each other to express biological functions. Because these multicomponent biological molecules weakly interact with each other, they can undergo regulatory dissociation and association upon inducing biological stimuli. To understand biological systems, we must, at first, aim to identify every possible complex co-existing in the given multicomponent system, and then quantitatively analyze the interactions of these complexed molecules. However, because of the complexity, it is hard to apply conventional analytical methods to analyze such multi-component equilibrium systems. We have developed a new analytical method that would enable us to perform structure and interaction analyses on multicomponent equilibrium systems.

Here, we will present the progress and development of the microfluidics-based, auto sampling equipment designed for continuous titration SAXS (Fig. 1). Using this newly designed equipment, we can automatically collect many scattering profiles while altering the molar ratios of each component involved in the multi-component equilibrium (Fig. 2); thus, enabling us to determine the system's free energy landscape of the multi-component equilibrium. Very recently, we successfully installed the sampling system into the beamline at the Photon Factory, Tsukuba, Japan, and performed titrationSAXS measurements on a signal transduction system containing Photoactive Yellow Protein (PBP) and it interaction partner (PBP). The SEC chromatography shows various complexes with different size exist in a mixed solution containing PYP and $\mathrm{PBP}$, indicating that this system is under a multi-component equilibrium state. The complexity prevents us from understanding the solution structure of each component. The continuous titration SAXS measurements allow us to extract scattering curves of at least three complexes and their concentration dependence (Fig. 2). Here, I would like to introduce the potential applications of this sampling system applied for continuous titration SAXS method in addition to the recent results we have obtained.

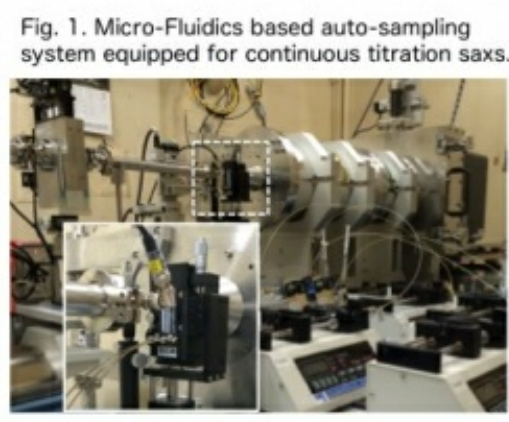

Fig. 3. Various complexes in mixed solution containing PYP and PBP

$\frac{1}{<}$

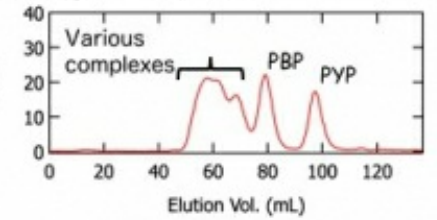

Fig. 2. Continuous titration SAXS using the auto-sampling system
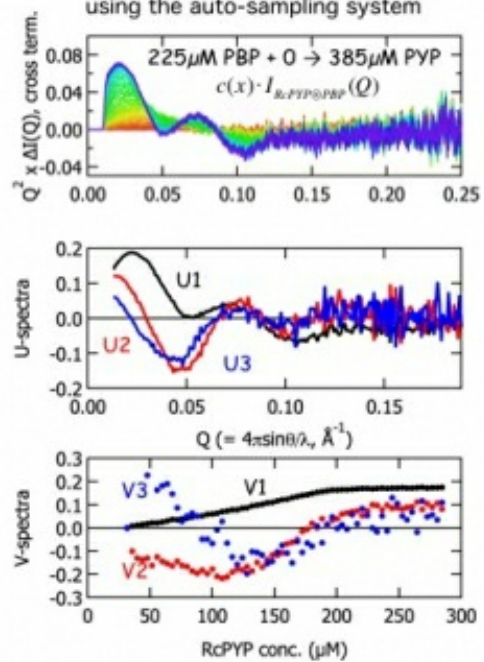

Keywords: Multi-component Equilibrium, Molecular systems, Photoactive Yellow Protein 